\title{
VEGF expression is augmented by hypoxia-induced PGIS in human fibroblasts
}

\author{
JIA WANG ${ }^{1,2,3^{*}}$, RYUJI IKEDA ${ }^{4 *},{\text { XIAO-FANG } \text { CHE }^{1}, \text { AKIO OOYAMA }^{5} \text {, MASATATSU YAMAMOTO }}^{1}$, \\ TATSUHIKO FURUKAWA ${ }^{1}$, KAZUHISA HASUI ${ }^{3}$, CHUN-LEI ZHENG ${ }^{6}$, YUSUKE TAJITSU ${ }^{4}$, \\ TOSHINORI OKA $^{5}$, SHO TABATA ${ }^{1}$, YUKIHIKO NISHIZAWA ${ }^{4}$, YOSHITO EIZURU ${ }^{7}$ and SHIN-ICHI AKIYAMA ${ }^{8}$ \\ ${ }^{1}$ Department of Molecular Oncology, Division of Cancer Genetics, Graduate School of Medical and Dental Science, \\ Kagoshima University, Kagoshima 890-8520; ${ }^{2}$ Department of Molecular Genetics, Medical Institute of Bioregulation, \\ Kyushu University, Fukuoka 812-8582; Departments of ${ }^{3}$ Immunology and ${ }^{4}$ Clinical Pharmacy and Pharmacology, \\ Graduate School of Medical and Dental Science, Kagoshima University, Kagoshima 890-8520, Japan; \\ ${ }^{5}$ Taiho Pharmaceutical. Co. Ltd, Personalized Medicine Research Laboratory; ${ }^{6}$ Department of Medical Oncology, \\ Cancer Hospital, Fudan University, Shanghai 200032, P.R. China; ${ }^{7}$ Division of Persistent and Oncogenic Viruses, \\ Center for Chronic Viral Diseases, Graduate School of Medical and Dental Science, Kagoshima University, \\ Kagoshima 890-8520; ${ }^{8}$ Department of Respiratory Medicine and Rheumatology, Institute of Health \\ Biosciences, The University of Tokushima Graduate School, Tokushima 770-8503, Japan
}

Received December 3, 2012; Accepted February 5, 2013

DOI: 10.3892/ijo.2013.1994

\begin{abstract}
Prostacyclin synthase (PGIS or PTGIS) is an enzyme that catalyses the conversion of prostaglandin $\mathrm{H}_{2}$ $\left(\mathrm{PGH}_{2}\right)$ to prostaglandin $\mathrm{I}_{2}\left(\mathrm{PGI}_{2}\right)$. $\mathrm{PGI}_{2}$ promotes cancer growth by activating peroxisome proliferator-activated receptor $\delta$ (PPAR $\delta$ ), and increases the expression levels of the pro-angiogenic factor vascular endothelial growth factor (VEGF). We found that the expression of the PGIS gene was enhanced in WI-38, TIG-3-20 and HEL human lung fibroblast cells and two cancer cell lines (NB-1 and G361) under hypoxic conditions. The main localization of PGIS changed from the cytoplasm to the nucleus by hypoxia in WI-38 cells. The induced PGIS had an enzymatic activity since the intracellular level of 6-keto-prostaglandin, a useful marker of $\mathrm{PGI}_{2}$ biosynthesis in vivo, was increased with the increasing levels of PGIS. Expression of VEGF was increased in parallel with PGIS induction under hypoxic conditions. PGIS knockdown resulted in the decreased expression of $V E G F$ mRNA. Since VEGF is a known PPAR $\delta$ target gene, we examined the effects of siRNAs targeting PPAR $\delta$ on the expression of VEGF under
\end{abstract}

Correspondence to: Dr Jia Wang, Division of Cancer Genetics, Department of Molecular Genetics, Medical Institute of Bioregulation, Kyushu University, Fukuoka 812-8582, Japan E-mail: wangjia0829jp@yahoo.co.jp

${ }^{*}$ Contributed equally

Key words: prostacyclin synthase, cancer-associated stromal fibroblasts, hypoxia, vascular endothelial growth factor, angiogenesis hypoxic conditions. Knockdown of PPAR $\delta$ suppressed the expression of VEGF under hypoxic conditions in WI-38 cells. These findings suggest that PGIS is induced by hypoxia and regulates the expression of $V E G F$ in fibroblasts. Fibroblasts in the hypoxic area of tumors may have an important role in tumor growth and angiogenesis.

\section{Introduction}

Cancer is subject to a unique microenvironment, composed of cancer cells, cancer-associated stromal fibroblasts (CAFs), endothelial cells, inflammatory cells and abundant extracellular matrix (1). It has been hypothesized that the components besides cancer cells in cancer tissues are functionally organized to promote survival of cancer cells against hypoxia in the host and generate a favorable microenvironment for cancer cells in both primary and metastatic sites (2). CAFs in the tumor stroma are involved in all stages of tumor development. A previous study has shown that fibroblasts from cancer mass produce $\mathrm{PGI}_{2}$ but fibroblasts from adjacent normal tissues do not (3). However, how CAFs are activated to produce $\mathrm{PGI}_{2}$ and if the activated CAFs can promote angiogenesis by generating VEGF itself are poorly understood.

We investigated the modulation of the gene expression in human fibroblasts under hypoxic condition using GeneChip analysis, and found that the expression of prostacyclin synthase (PGIS) was upregulated. PGIS, a membrane-bound heme protein with spectral characteristics of cytochrome $\mathrm{p} 450$ (CYP), is also an enzyme which catalyzes the conversion of prostaglandin $\mathrm{H}_{2}\left(\mathrm{PGH}_{2}\right)$ to form prostacyclin $\left(\mathrm{PGI}_{2}\right)$. PGIS is localized to the microsomal fractions of platelets, vascular endothelial cells, and vascular smooth muscle cells (4-8). 
PGIS-deficient mice generated by gene targeting are associated with thickening of arterial walls, interstitial fibrosis with nephrosclerosis and kidney infarction (9). Interestingly, some groups reported that $\mathrm{PGI}_{2}$, the product of PGIS, promotes colorectal cancer growth probably by activating peroxisome proliferator-activated receptor $\delta$ (PPAR $\delta)(10)$. Inhibition of COX-2-derived prostacyclin $\left(\mathrm{PGI}_{2}\right)$ induces colon cancer cell apoptosis (11). $\mathrm{PGI}_{2}$ also regulates the transcription of VEGF by PPAR $\delta(12,13)$.

In the present study, we focused our study on PGIS and confirmed that the expression of PGIS was upregulated in human fibroblasts under hypoxic condition and the induced PGIS was involved in the induction of VEGF. We have suggested that PPARS enhances the hypoxic induction of VEGF.

\section{Materials and methods}

Antibodies. The polyclonal antibody against PGIS was purchased from Cayman. The polyclonal antibody against Erk-1 was purchased from Santa Cruz Biotechnology (Santa Cruz, CA). The monoclonal antibody against GAPDH was purchased from Ambion Inc. (Austin, TX).

Patients. The paraffin specimens of 3 cases of colon cancer were supplied from Department of Surgical Oncology Kagoshima University Faculty of Medicine. This study was performed with the approval of the Ethics Committee for Epidemiological Studies in Kagoshima University Graduate School of Medical and Dental Sciences.

Cell lines and cell cultures. WI-38 and TIG-3-20 human fibroblasts were purchased from Health Science Research Resources Bank (HSRRB), human embryonic lung fibroblasts (HEL) and human neuroblastoma cells (NB-1) cell lines were provided from Professor Y. Eizuru (Kagoshima University, Kagoshima, Japan) and Professor A. Tomoda (Tokyo Medical University, Tokyo, China), respectively. Other cell lines were stocked in our laboratory. All of the cells were cultured in Dulbecco's modified Eagle's medium (DMEM) containing 10\% FCS, 2 mM glutamine and $100 \mathrm{U} / \mathrm{ml}$ penicillin at $37^{\circ} \mathrm{C}$ in a $5 \% \mathrm{CO}_{2}$ humidified atmosphere. Hypoxic condition was induced by incubating the cells in a Personal Multigas Incubator (Astec) at $37^{\circ} \mathrm{C}$ with humidified $1 \% \mathrm{O}_{2}$.

GeneChip analysis. cDNA and biotinylated cRNA were synthesized according to the standard protocols provided by Affymetrix (Santa Clara, CA). Biotinylated cRNA were purified using the Sample Cleanup Module (Affymetrix), and subsequently hybridized, according to the manufacturer's standard protocols, to Affymetrix HGU133A GeneChips (which contain 22283 probe sets). Arrays were scanned using an Affymetrix confocal laser scanner. A gene expression analysis software program, GeneSpring, version 7.1 (Agilent), was used to perform statistical analysis.

Reverse transcription-PCR. $1 \mu \mathrm{g}$ first-strand cDNA was used for each polymerase chain reaction (PCR). The human PGIS primers were as follows: forward, 5'-ctggctcctgc tcttccttctcaag-3'; reverse, 5'-caccacgtcgcaggttgaattcttg-3'. The human $V E G F$ primers were: forward, 5'-tgtcttgggtgcattggag cettgc-3'; reverse, 5'-gttgtgctgtaggaagctcatctctc-3'. The human GAPDH primers for internal control were: forward, 5'-tgcacc accaactgcttag-3'; reverse, 5'-gaggcagggatgatgttc-3'. The human PPAR $\delta$ primers were: forward, 5'-actgagttcgccaagagcatc-3'; reverse, 5'-aacgttcatgaggectggccg-3'.

The PCR amplification mixture was adjusted to a final volume of $20 \mu \mathrm{l}$. Twenty-eight cycles were performed at $94^{\circ} \mathrm{C}$ for $30 \mathrm{sec}$ for denaturation, $58^{\circ} \mathrm{C}$ for $30 \mathrm{sec}$ for annealing, and $72^{\circ} \mathrm{C}$ for $30 \mathrm{sec}$ for extension. The PCR products were separated on $1.5 \%$ agarose gel and then stained with ethidium bromide.

Real-time reverse transcription-PCR quantification. Total cellular RNA was extracted using TRIzol reagent according to the manufacturer's instructions (Invitrogen, Carlsbad, CA). One microgram of RNA was reverse transcribed using a firststrand cDNA synthesis kit (ReverTra Ace- $\alpha-$, Toyobo, Osaka, Japan). The set of SYBR Premix Ex Taq kit was purchased from Takara Bio (Shiga, Japan). Human PGIS, VEGF, GAPDH gene expression levels were assayed by real-time reverse PCR (PRISM 7900HT, Applied Biosystems, Foster City, CA) according to the technical brochure of the company. Human $G A P D H$ was used for normalization. Quantification of target gene expression was obtained with the comparative cycle threshold method according to the instructions of the manufacturer (Applied Biosystems). All experiments were performed in triplicate for each data point. Each quantitation was performed with the standard curve method.

Protein extraction and immunoblot analysis. Cells were harvested and lysed with RIPA buffer [50 mM Tris-HCI (pH 7.4), $150 \mathrm{mM} \mathrm{NaCl}, 1 \mathrm{mM}$ EGTA, $1 \mathrm{mM}$ EDTA, $20 \mathrm{mM} \mathrm{NaF}, 100 \mathrm{mM} \mathrm{Na}_{3} \mathrm{VO}_{4}, 0.5 \%$ Nonidet P-40 (NP-40), $1 \%$ Triton X-100, 1 mM PMSF, DTT, aprotinin, APMSF and protease inhibitor cocktail (Sigma)]. The lysates were passed through a 21-gauge needle to break up the cell aggregates and were cleared by centrifugation at $14,000 \mathrm{x}$ g for $15 \mathrm{~min}$ at $4^{\circ} \mathrm{C}$; the supernatant (total cell lysate) was immediately used or stored at $-80^{\circ} \mathrm{C}$ until use.

Lysates were subjected to $9.4 \%$ SDS-polyacrylamide gel electrophoresis (SDS-PAGE), and then were transferred to polyvinylidene difluoride membranes (Immobilon-P transfer membrane; Millipore, Bedford, MA). The membrane was treated with buffer A [350 mM NaCl, $10 \mathrm{mM}$ Tris- $\mathrm{HCl}$ (pH 8.0), and $0.05 \%$ Tween-20] containing 3\% skimmed milk for $1 \mathrm{~h}$ and incubated with the indicated antibody $(1: 1,000)$ in buffer $\mathrm{A}$ containing $3 \%$ skimmed milk for 1 hour. Following four washes with buffer A (10 min each), the membrane was incubated with a peroxidase-conjugated horse anti-mouse IgG diluted 1:1,000 in buffer A containing 3\% skimmed milk for 1 hour. The membrane was washed with buffer A and developed using the enhanced chemiluminescence western blot analysis detection system (Amersham Biosciences, Buckinghamshire, UK).

Subcellular fractionation. Cells were washed with PBS and then resuspended in 3 volumes of buffer $B$ [10 mM HEPES-KOH (pH 7.8), 10 mM KCl, 0.1 mM EDTA (pH 8.0), $1 \mu \mathrm{g} / \mathrm{ml}$ aprotinin and $1 \mathrm{mM}$ p-aminophenyl methanesulfonyl fluoride]. The cells were disrupted by dounce homogenizer. The nuclear fractions were centrifuged at 13,000 rpm for $5 \mathrm{~min}$ at $4^{\circ} \mathrm{C}$. The supernatant was used as cytosolic fractions. 
Table I. GeneChip analysis of the modulation of the gene expression in fibroblasts under hypoxic condition.

\begin{tabular}{llr}
\hline Gene symbol & \multicolumn{1}{c}{ Description } & Fold change MAX \\
\hline AK3 & Adenylate kinase 3-like 1 & 3.2 \\
BNIP3 & BCL2/adenovirus E1B 19 kD-interacting protein 3 & 4.9 \\
CYR61 & Cysteine-rich, angiogenic inducer, 61 & 2.3 \\
FAM13A1 & Family with sequence similarity 13, member A1 & 5.9 \\
FGF1 & Fibroblast growth factor 1 (acidic) & 3.0 \\
FGFR1 & Fibroblast growth factor receptor 1 & 3.7 \\
IGFBP3 & Insulin-like growth factor-binding protein & 26.6 \\
MBNL1 & Muscleblind-like (Drosophila) & 3.0 \\
MYH10 & Myosin, heavy polypeptide 10, non-muscle & 3.4 \\
NDRG1 & Homo sapiens N-myc downstream regulated gene 1 & 8.5 \\
PDGFC & Platelet derived growth factor C & 7.2 \\
PGK1 & Phosphoglycerate kinase 1 & 2.3 \\
PTGIS/PGIS & Homo sapiens prostaglandin I (prostacyclin) synthase & 13.5 \\
STC1 & Stanniocalcin 1 & 3.9 \\
VEGF & Vascular endothelial growth factor & 5.8 \\
WISP1 & WNT1 inducible signaling pathway protein 1 & 9.9 \\
XDH & Xanthine dehydrogenase & 60.1 \\
\hline
\end{tabular}

The increased levels of some genes that are closely related to tumor growth, metastasis, endothelial cell biology, and TGF- $\beta$ signaling pathway are shown.

Inhibition of PGIS and PPAR expression by small interfering $R N A$. Specific small interfering RNA (siRNA) of PGIS was purchased from Santa Cruz Biotechnology. SiRNA duplexes of $P P A R \delta$ were synthesized using the Silencer ${ }^{\mathrm{TM}}$ siRNA construction kit (Ambion Inc.). The siRNA used in this study consisted of a 21-nucleotide sense strand and a 21-nucleotide antisense strand with a two-nucleotide overhang at 3'-end. The sequences were: PPAR $\delta$-siRNA(1) sense 5'-CAUGGAGUGCCGGGUGUG CUU-3' starting at nucleotide 211 from AUG start codon of human PPAR $\delta$ coding sequence. PPAR $\delta$-siRNA(1) antisense, 5'-GCACACCCGGCACUCCAUGUU-3'. PPARס-siRNA(2) sense 5'-GCUGGAGUACGAGAAGUGUUU-3' starting at nucleotide 313 from AUG start codon of human PPAR $\delta$ coding sequence. PPAR $\delta$-siRNA(2) antisense, 5'-ACACUUCUCG UACUCCAGCUU-3'.

Transfections were accomplished using Lipofectamine 2000 (Invitrogen), according to the manufacturer's protocol. After transfection, the cells were incubated under normoxic condition for $5 \mathrm{~h}$, then changed with fresh DMEM and incubated under hypoxic condition until harvested at different time points. The effect of the siRNA on the expression of PGIS and VEGF was assessed using RT-PCR and real-time PCR, as described above.

6-Keto-prostaglandin Fla enzyme immunoassay. 6-Ketoprostaglandin F1 $\alpha$ (stable hydrolysis product of $\mathrm{PGI}_{2}$ ) was analyzed by specific enzyme immunoassay kit (EIA) (Assay Designs), following the manufacturer's instructions. Lysates were added to every well of the kit plate. The resulting values of 6-keto-prostaglandin F1 $\alpha$ concentrations in the supernatant were normalized for lyses buffer.
Confocal fluorescence microscopy. WI-38 and HEL cells were cultured under normoxic or hypoxic condition for different time, and fixed with $4 \%$ paraformaldehyde (PFA) in PBS for $30 \mathrm{~min}$ at room temperature. After blocking with $5 \%$ normal goat serum in PBS/0.02\% Tween-20, the cells were incubated with an antibody against PGIS (1:100) at $4^{\circ} \mathrm{C}$ overnight. After washing thrice in PBS/0.02\% Tween-20, the cells were incubated with secondary antibodies (antirabbit-FITC $1 \times 100)$. Nuclei were stained by incubating cells with $6 \mu \mathrm{mol} / 1$ 4'6-diamidino-2-phenylindole (DAPI). The cells were observed using confocal fluorescence microscopy (FV500, Olympus Corporation).

Immunohistochemistry. Immunohistochemical studies for PGIS and HIF-1 $\alpha$ were performed on formalin-fixed, paraffin-embedded surgical sections obtained from patients with colorectal cancer. Slides were deparaffinized in xylene and rehydrated in a graded alcohol series. To retrieve antigen, sections were heated in the citrate buffer pH 6.0 (Target retrieval solution, S1699, Dako, Carpinteria, $\mathrm{CA}$ ) for $5 \mathrm{~min}$ at $121^{\circ} \mathrm{C}$ by means of an autoclave. For detection and visualization, the retrieved antigens were reacted with the appropriate specific primary antibodies solution (diluted to 1:100) for $1 \mathrm{~h}$ and were visualized by means of a polymer method (ChemMate Envision, K5027, Dako) and colorimetric diaminobenzidine (DAB)-peroxide $\left(\mathrm{H}_{2} \mathrm{O}_{2}\right)$ reaction (DAB+ liquid system, K3468, Dako). Nuclear counterstaining was hematoxylin (ChemMate hematoxylin, S2020, Dako). These procedures were performed by an autostainer (Autostainer, Dako) with rinsing buffer warmed at $35^{\circ} \mathrm{C}$ (14). 
A
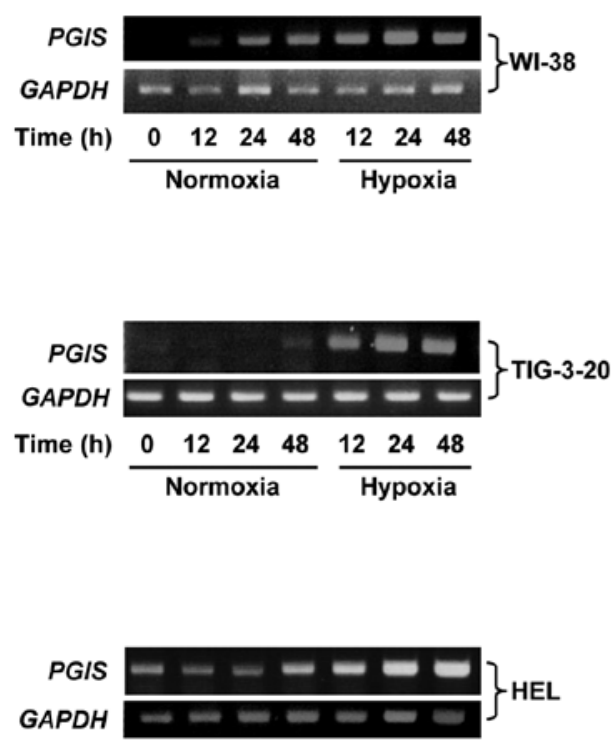

Time (h) \begin{tabular}{lllllll}
24 & 48 & 3 & 6 & 12 & 24 & 48 \\
\hline
\end{tabular}
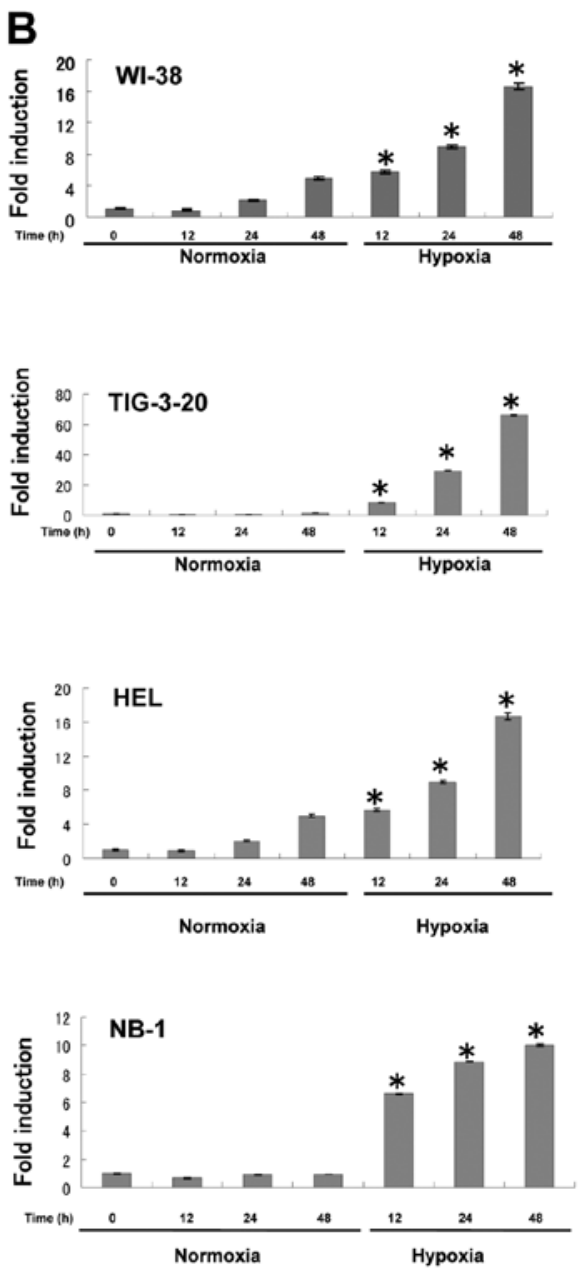

Normoxia

Hypoxia
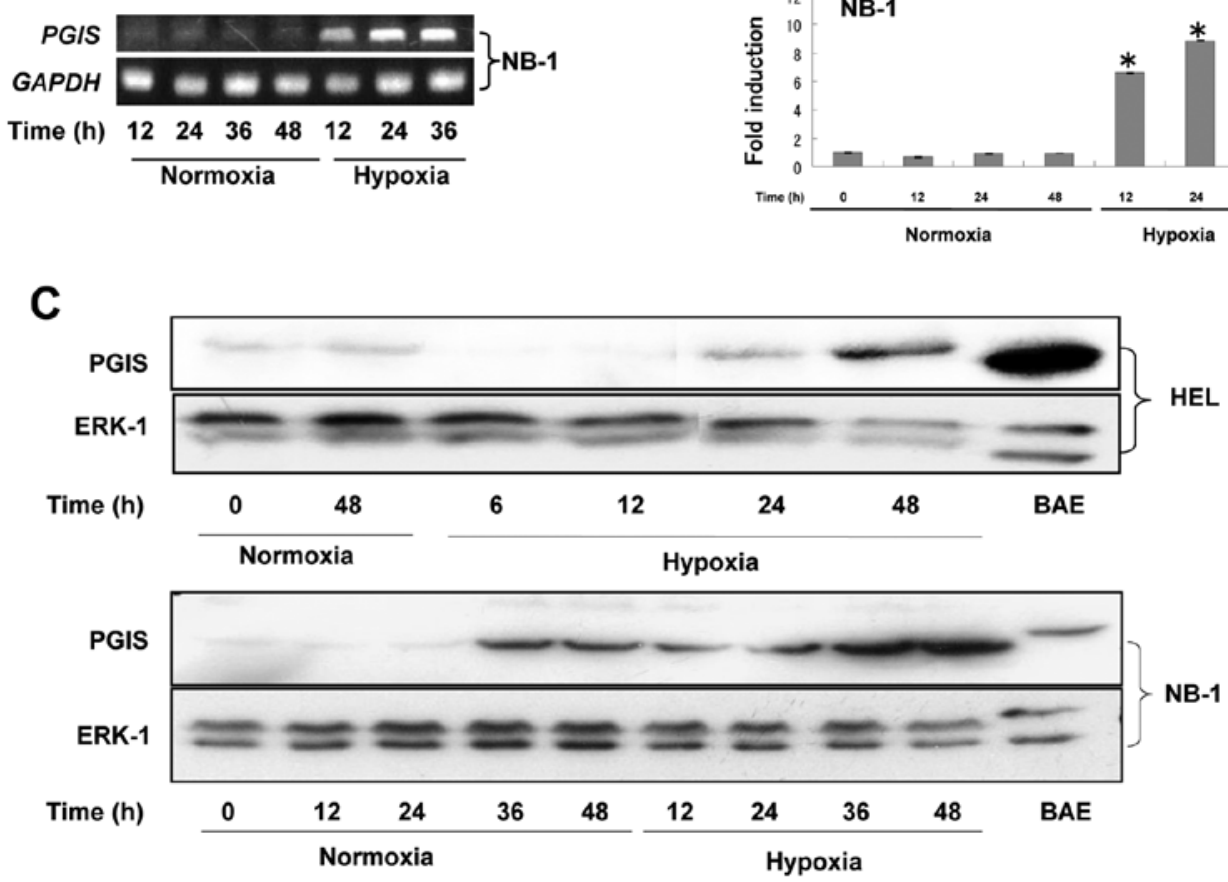

Figure 1. (A) RT-PCR and (B) real-time PCR analysis showed the expression levels of PGIS were time-dependently increased in fibroblasts (WI-38, TIG-3-20 and HEL) and cancer cells (NB-1) under hypoxia. GAPDH was used for internal control. Time-course was sustained until $48 \mathrm{~h}$ ("p<0.01 vs cells exposed to normoxia). (C) The expression levels of PGIS protein in fibroblasts (HEL cells) and neuroblastoma cells (NB-1) were enhanced time-dependently under hypoxic condition. ERK-1 was used for normalization. Cells were incubated until $48 \mathrm{~h}$ after exposure to hypoxia.

\section{Results}

GeneChip analysis. GeneChip analysis was used for identifying the modulation of the gene expression in fibroblasts under hypoxic condition. A total of 234 genes were differentially expressed between fibroblasts under normoxic or hypoxic condi- tion in this study. Relative to normoxic condition data profiling, 172 genes were upregulated and 62 genes were downregulated (3-fold change). Of 172 upregulated genes, genes related to tumor metastasis, endothelial cell biology and TGF- $\beta$ signaling pathway etc. were included. Table I lists some upregulated genes in fibroblasts under hypoxic condition. Among these genes, we 
A
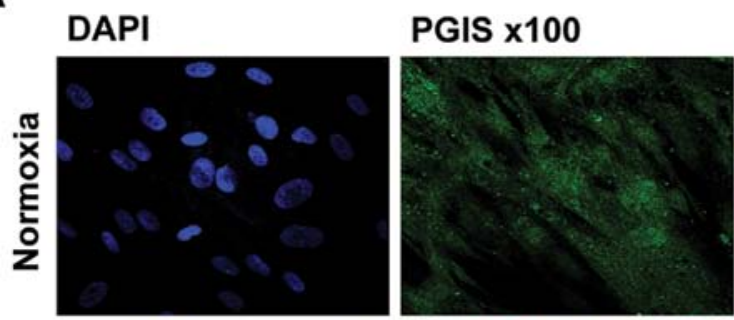

Merge
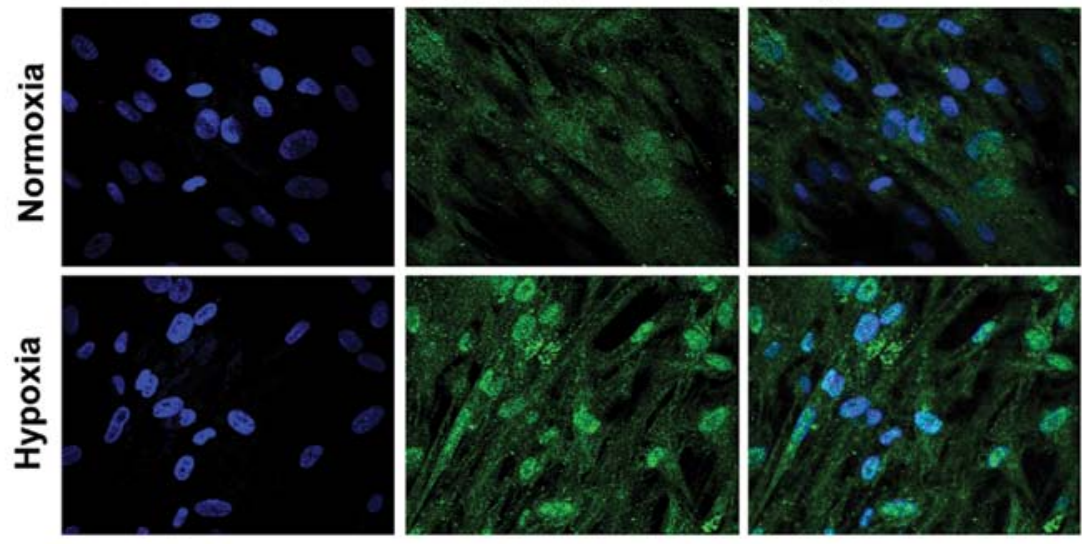

B

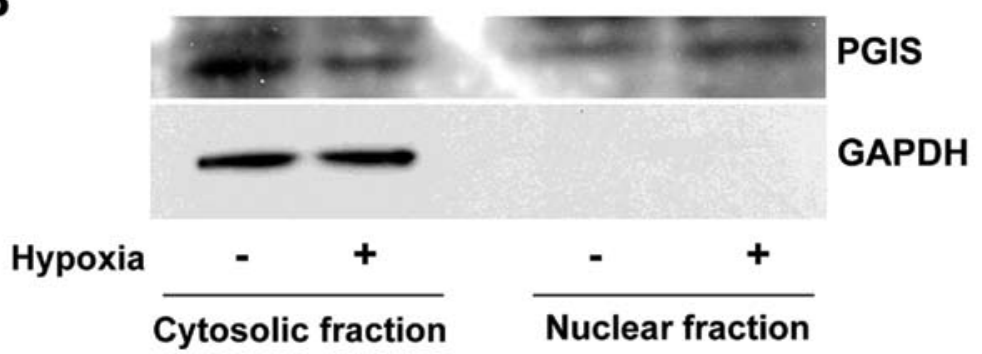

Figure 2. (A) Confocal microscopy shows the distribution of PGIS in the cytoplasm and nuclei of fibroblasts (WI-38) when incubated under normoxic condition. Under hypoxic condition, expression of PGIS was augmented and translocated to nuclei. (B) The levels of PGIS in cytoplasm and nuclei under normoxic or hypoxic conditions. The cells were incubated under normoxic or hypoxic conditions for $48 \mathrm{~h}$. Cytoplasmic or nuclear fractions were prepared and subjected to immunoblot analysis with a specific antibody against PGIS.

focused on PGIS, which showed 13.5-fold increased expression levels.

Expression of $m R N A$ for PGIS proteins determined by RT-PCR or real-time qualitative $P C R$. As shown in Fig. 1A, the expression levels of $P G I S$ gene were significantly enhanced in fibroblasts such as WI-38, TIG-3-20 and HEL time-dependently under hypoxic condition. To quantitate the mRNA expression levels of $P G I S$ in fibroblasts, real-time quantitative RT-PCR was performed (Fig. 1B). Moreover, the expression levels of PGIS in some human cancer cells were also investigated in this study. The time-dependently enhanced expression levels of PGIS under hypoxic condition were detected in human neuroblastomas (NB-1) (Fig. 1A and B) and human melanomas (G361), but not in glioblastomas (U251MG), pancreas cancer (MiapacaII), non-small cell lung cancer (A549), KB/TP and EJ/TP cells (15) (data not shown). PGIS mRNA was not detected in human macrophages (U937 and THP1) under normoxic or hypoxic condition (data now shown). Since the enhanced expression levels of PGIS were not detected in fibroblasts cultured in the medium containing 1\% FCS (data not shown), the induction of PGIS could not be attributed to the low nutrient condition. These results suggested that the enhanced expression of PGIS mRNA in fibroblasts and some cancer cells was induced by hypoxic condition.
Expression and intracellular localization of PGIS in fibroblasts and neuroblastomas. We next examined expression levels of PGIS in fibroblasts (HEL) and neuroblastomas (NB-1) by western blot analysis (Fig. 1C). Expression levels of PGIS protein in both cell lines were also enhanced time-dependently under hypoxic condition consistent with mRNA level. To identify the subcellular localization of PGIS induced by hypoxia, PGIS was observed using confocal fuorescence microscopy. As shown in Fig. 2A, the expressions of PGIS were mainly localized in the cytoplasm of WI-38 cells, and translocated to nuclei under hypoxic condition. Immunoblot analysis also showed that PGIS was translocated from cytoplasm to nuclei when the cells were cultured under hypoxic condition (Fig. 2B).

The effect of PGIS knockdown on the induction of VEGF under hypoxic condition in fibroblasts. $\mathrm{PGI}_{2}$ is known to increase the expression levels of the pro-angiogenic factor VEGF $(16,17)$. To confirm that PGIS is involved in the enhanced expression of VEGF under hypoxic condition in fibroblasts, $P G I S$ siRNA was used to knockdown the expression of PGIS. The induction of PGIS mRNA expression by hypoxia was considerably suppressed by PGIS siRNA (Fig. 3A). PGIS knockdown resulted in the decreased expression of $V E G F$ mRNA (Fig. 3B). These results show that PGIS is required for the hypoxic induction of VEGF. 
A

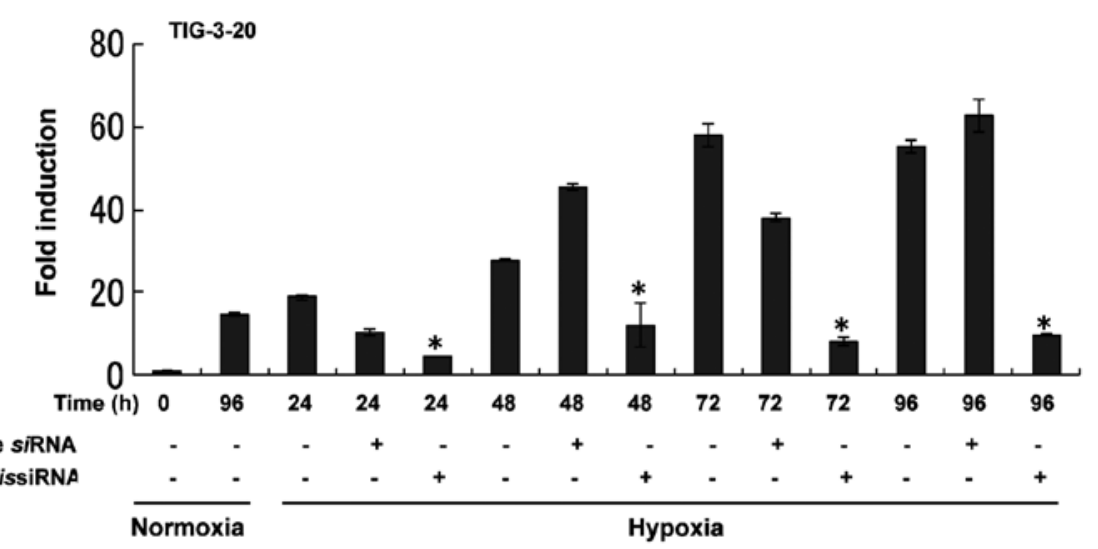

B

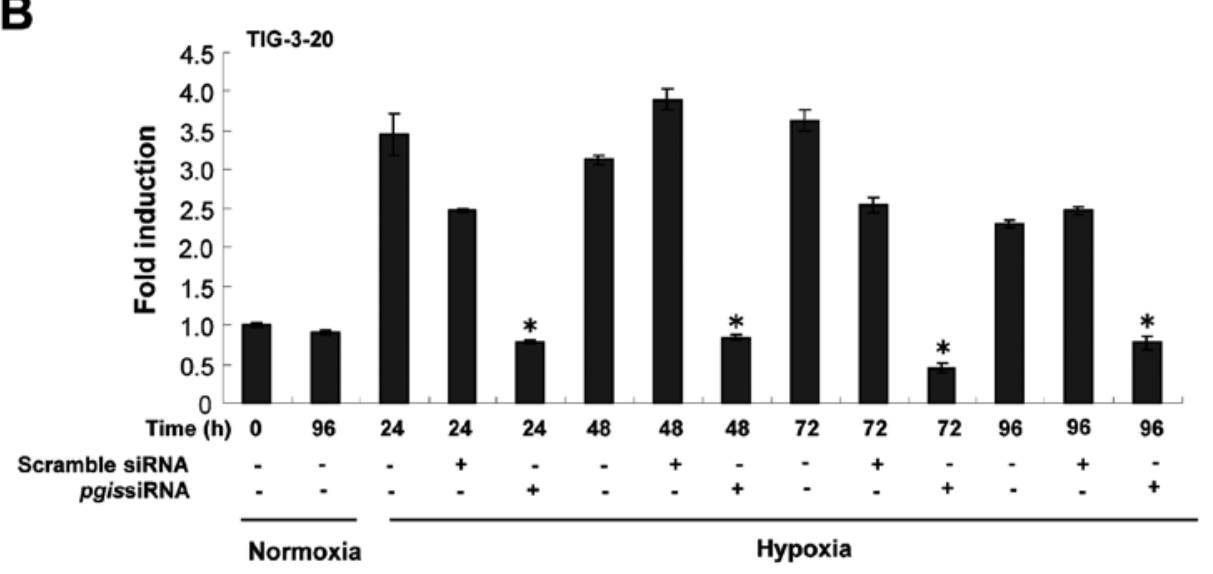

C
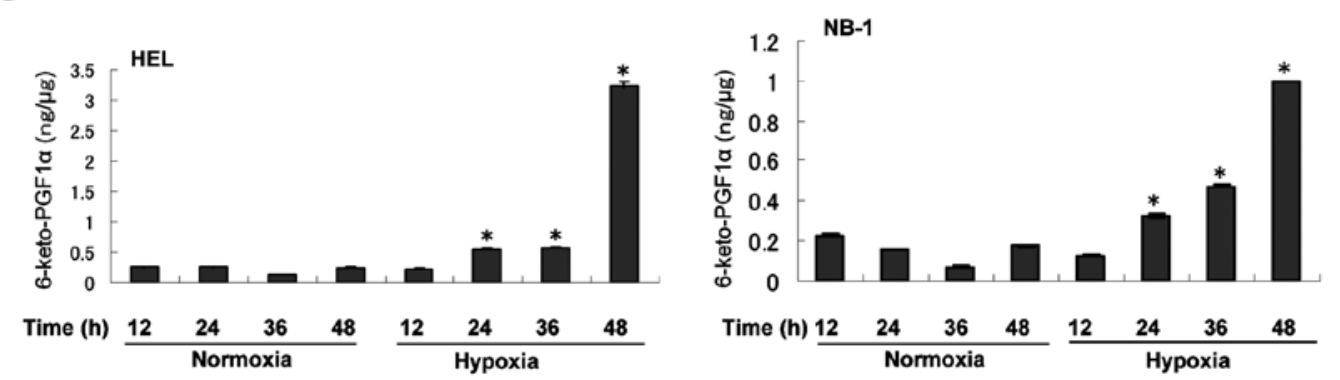

Figure 3. Downregulation of (A) PGIS and (B) VEGF mRNA levels by PGISsiRNA in TIG-3-20 cells under hypoxic condition. Knockdown of $P G I S$ attenuated $V E G F$ expression under hypoxic condition until $96 \mathrm{~h}$ (siRNA $100 \mathrm{nM}$; ${ }^{*} \mathrm{p}<0.05$ ). (C) Levels of 6-keto-prostaglandin in HEL and NB-1 under hypoxic condition. In HEL and NB-1, PGIS activity was measured by determining the production of 6-keto-prostaglandin F1 $\alpha$, a degradation product of $\mathrm{PGI}_{2}$. When cells were incubated under hypoxic condition, the PGIS activity was also increased in a time-dependent manner. The PGIS activity in HEL or NB-1 cells under hypoxic condition for 36 and $48 \mathrm{~h}$ was significantly increased compared with that under normoxic condition ( $\mathrm{p}<0.05)$.

Expression of 6-keto-prostaglandin Fla in HEL and NB-1 cells under hypoxic condition. Since $\mathrm{PGI}_{2}$ is unstable, the level of the stable hydrolysis metabolite of $\mathrm{PGI}_{2}$, 6-keto-prostaglandin $\mathrm{F} 1 \alpha$ was analyzed in HEL and NB-1 cells under hypoxic condition. The level of 6-keto-prostaglandin $\mathrm{F} 1 \alpha$ was also significantly increased in a time-dependent manner under hypoxic condition compared with that under normoxic condition $(\mathrm{p}<0.05$, triplicate determinations; Fig. 3C).

The effect of PPARd-targeting siRNAs on the expression of $V E G F$ under hypoxic condition. It was previously reported that endogenous $\mathrm{PGI}_{2}$ generation by co-expression of COX-2 and PGIS activated PPARS (18). Because the expression of COX-2 was not induced under hypoxic condition in fibroblasts and tumor cells (data not shown), we considered that $\mathrm{PGI}_{2}$ produced by the hypoxia-induced PGIS entered the nuclei and interacted with PPARS.

To examine whether PPAR $\delta$ regulates the expression of VEGF, WI-38 cells transfected with PPAR $\delta$ siRNA or green fluorescent protein (GFP) siRNA as a control were cultured under hypoxic condition. As shown in Fig. 4, downregulation of PPAR $\delta$ suppressed the expression of $V E G F$ mRNA. These 


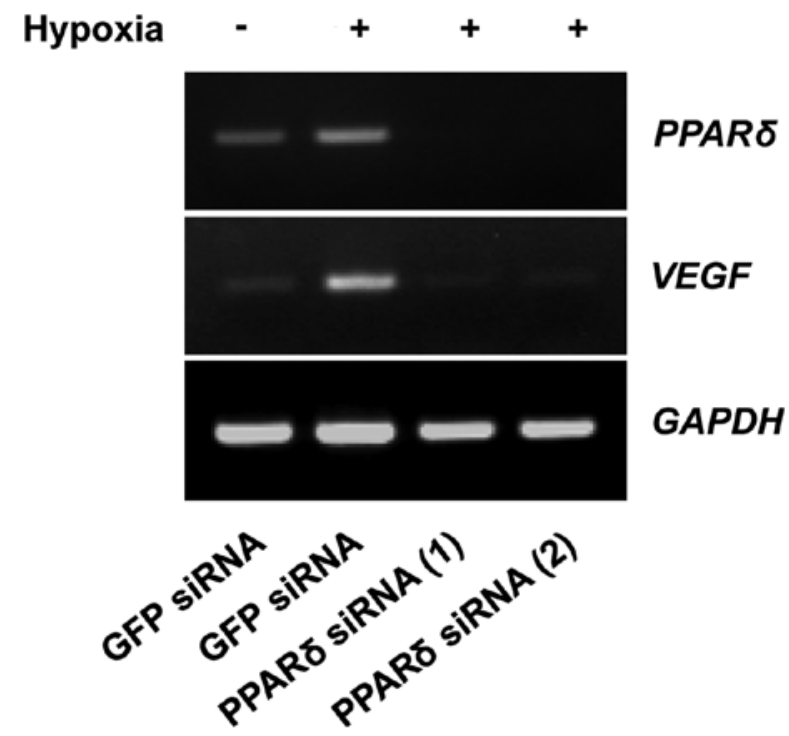

Figure 4. Effects of siRNA-mediated silencing of PPAR $\delta$ or HIF-1 $\alpha$ on the expression of VEGF in WI-38 cells. WI-38 cells transfected with siRNA targeting $P P A R \delta$ or $G F P$ were cultured under hypoxic condition for $24 \mathrm{~h}$. Total RNA was extracted and the expressions of $P P A R \delta, V E G F$ and $G A P D H$ were evaluated by RT-PCR. results indicate that $P P A R \delta$ regulates transcription of $V E G F$ in WI-38 cells.

Expression of PGIS in clinical patients by immunohistochemistry. To determine whether PGIS is expressed in fibroblasts and cancer cells in clinical human tumor tissues, we examined 3 cases of human colon cancer tissues. As shown in Fig. 5, PGIS was expressed in cancer cells and fibroblasts which are located in the deep layers of the intestinal wall but not in the surface layers (Fig. 5a-b). Immunohistochemical staining of PGIS validated the induced expression of PGIS in fibroblasts under hypoxic condition in tumors (Fig. 5d).

\section{Discussion}

In this study, we observed the induction of PGIS expression under hypoxic condition, and confirmed that the induced PGIS was, at least in part, responsible for the increased expression of VEGF in human fibroblasts. We showed that PGIS was induced in fibroblasts and cancer cells by hypoxia, but not in cells under low nutrient condition (Fig. 1).

PGIS was accumulated in the nuclei of fibroblasts under hypoxia, and the amount of 6-keto-PGF ${ }_{1 \alpha}$ was increased with
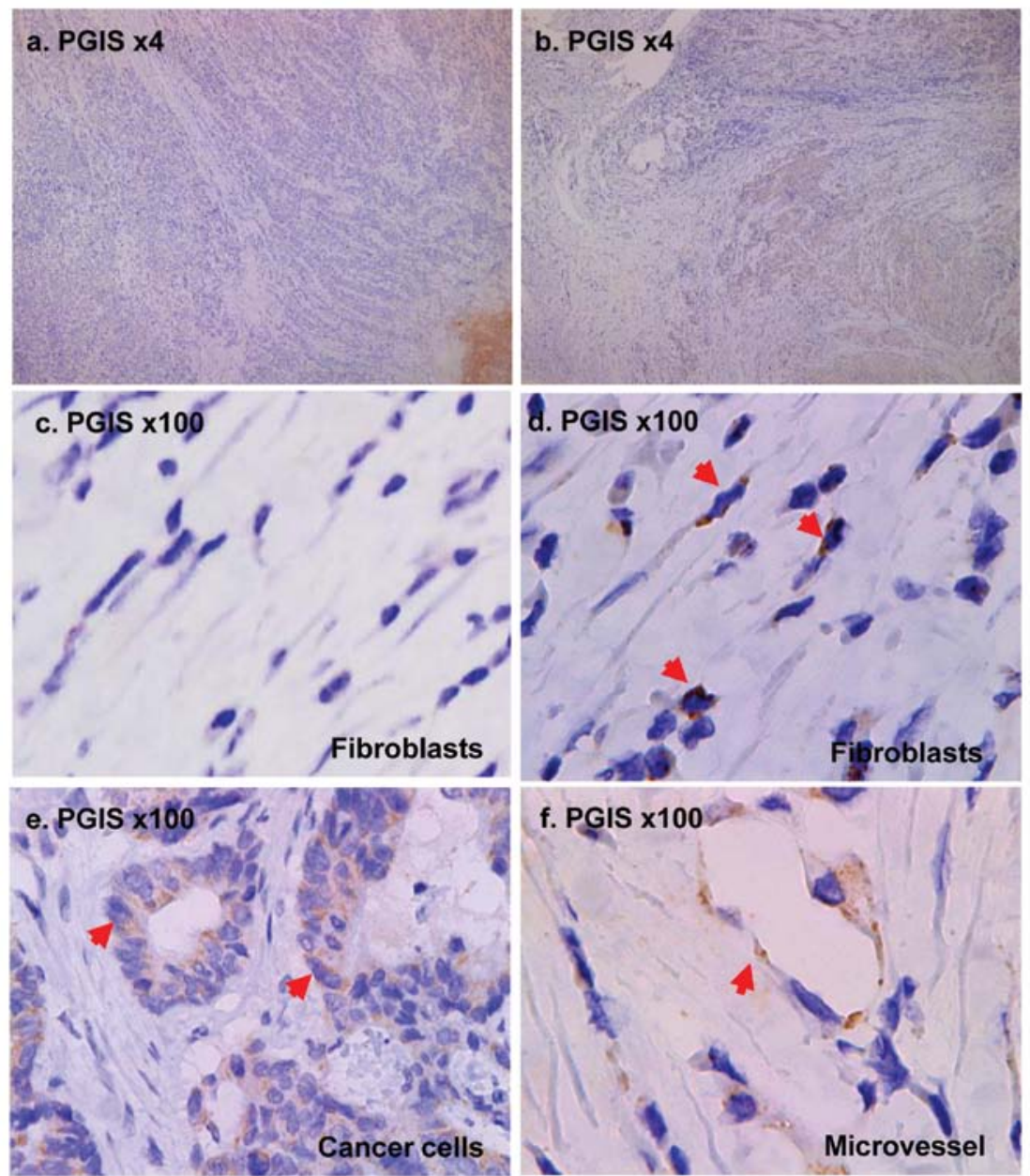

Figure 5. Immunohistochemical staining for (a-f) PGIS in human colon cancer sections. (e) Colon cancer cells and (d) fibroblasts which are located around cancer cells expressed PGIS in the deep layers of the (b) intestine wall but not the (a and c) surface layers. The PGIS was also expressed in (f) epithelial cells of microvessels. (Arrowhead shows PGIS positive cells). 


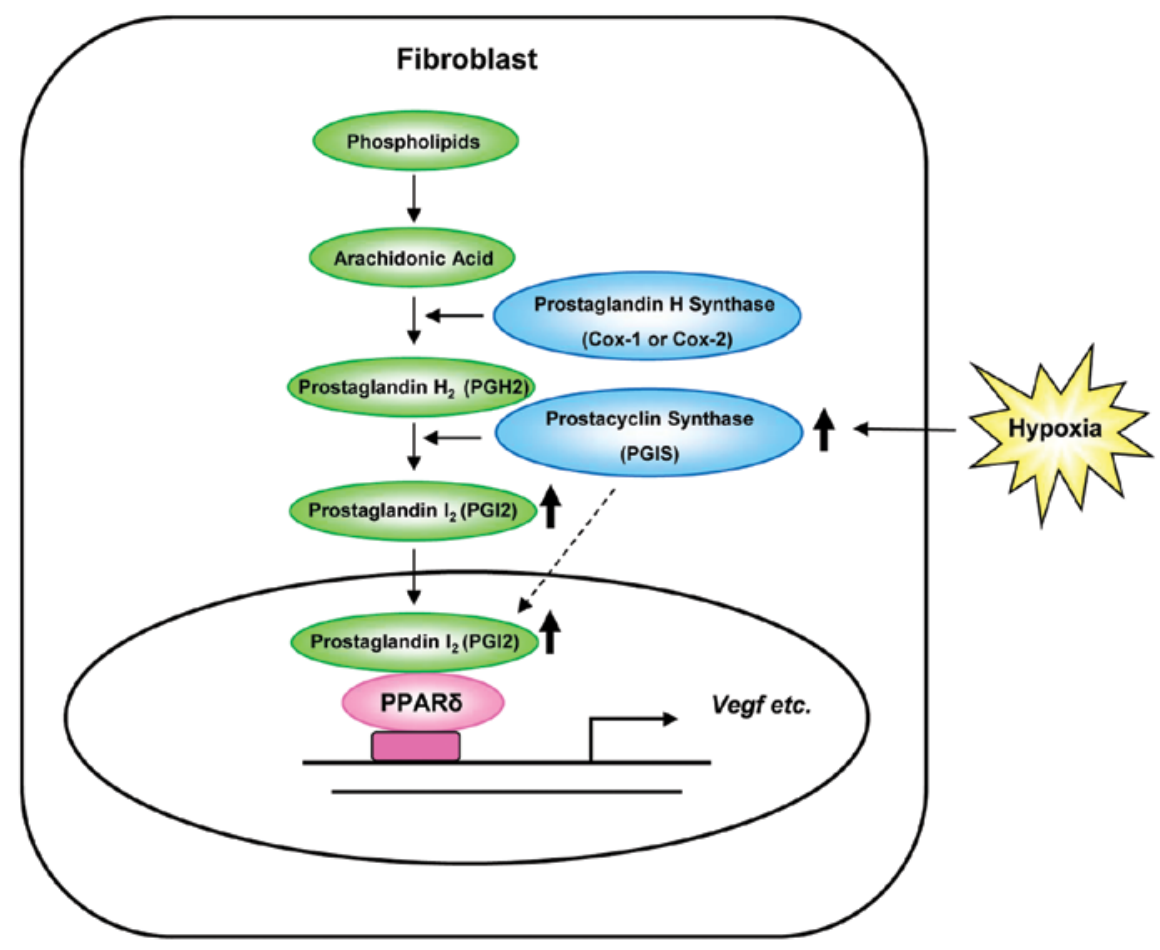

Figure 6. Schematic pathway for hypoxia-induced VEGF expression via $\mathrm{PGI}_{2}$ in human fibroblasts. In the pathway for prostaglandin synthesis, an enzyme named PGIS catalyses the conversion of $\mathrm{PGH}_{2}$ to $\mathrm{PGI}_{2}$. Our study showed that the level of PGIS in nuclei was enhanced in human fibroblasts under hypoxic condition, and suggested that increased $\mathrm{PGI}_{2}$ binds to PPAR $\delta$ to augment the transcription of its target genes such as Vegf.

increasing expression of PGIS, suggesting that $\mathrm{PGI}_{2}$ was produced by the induced PGIS.

We considered that the increased $\mathrm{PGI}_{2}$ was caused by the hypoxia-induced PGIS, since the expression of COX-2 was not induced under hypoxic condition in both fibroblasts and tumor cells (data not shown). PGI $_{2}$ has been shown to be a ligand for peroxisomal-proliferator-activated receptors (PPAR). It was previously reported that endogenous $\mathrm{PGI}_{2}$ generation by co-expression of COX-2 and PGIS led to transcriptional activation of PPAR $\delta$ (18). Increased PPAR $\delta$ augmented the transcription of its target genes such as VEGF (19), $\mathrm{PGI}_{2}$ is also known to increase the expression of VEGF in cultured lung fibroblasts (17). $\mathrm{PGI}_{2}$ seems to play an important role in generating VEGF and consequently angiogenesis and tumor growth. Therefore, we investigated VEGF expression in fibroblasts under hypoxic condition. VEGF was time-dependently induced under hypoxic condition in a manner similar to PGIS. Downregulation of PGIS suppressed the increased expression of VEGF under hypoxic condition, indicating that PGIS is involved in the regulation of VEGF expression under hypoxic condition (Figs. 3A, B and 4). These results suggest that PGIS locates upstream of the VEGF gene in agreement with previous results $(12,13)$.

The pro-angiogenic factor VEGF overexpressed in most cancer cells is part of the system that restores oxygen supply to tumor tissues when blood circulation is inadequate (20). It is generally accepted that VEGF recruited by cancer cells acts on cancer mesenchyme-like endothelial and inflammatory cells to cause angiogenesis. VEGF induced by PGIS in fibroblasts under hypoxic condition may play an important role in the progression of carcinomas.
Moreover, the main localization of PGIS changed from cytoplasm to nuclei by hypoxia in human fibroblast cells (Fig. 2). This finding suggested that PGIS itself may enter the nuclei, where it synthesizes $\mathrm{PGI}_{2}$ to regulate the expression of VEGF (Fig. 6). The mechanism of this nuclear translocation of PGIS remains to be uncovered.

Finally, the immunostaining patterns of tumors from clinical cancer patients were in accord with the data obtained from in vitro experiments (Fig. 5). The PGIS-positive cancer cells and fibroblasts were only detected in the deep layers of the intestinal wall where is thought to be deficient in oxygen but not in the surface layers. These findings might support the view that PGIS is involved in tumor progression and angiogenesis under hypoxic condition. $\mathrm{PGI}_{2}$ was previously shown to activate PPARס leading to subsequent acceleration in intestinal tumor growth in $\mathrm{Apc}^{\mathrm{Min} /+}$ mice (21). Meanwhile, $\mathrm{PGI}_{2}$-mediated activation of PPAR $\delta$ was implicated in negative growth of lung cancer cell lines (22). The role of PGIS in tumor growth might be different in respective cells.

Transcriptional regulation of the PGIS gene has rarely been reported in the literature. Recently, Camacho et al defined the minimal PGIS promoter region responsive to hypoxia. However, it did not contain a putative hypoxia responsive element (HRE). Furthermore, they observed that knockdown of HIF-l $\alpha$ abrogated hypoxia-induced PGIS upregulation in human vascular cells, and suggested that PGIS transcriptional activity enhanced by hypoxia could be the results of the cooperative binding of several transcription factors to PGIS promoter (23). Further study is needed to elucidate the molecular basis for the induction of PGIS by hypoxia. 
In conclusion, this study provides new and important information about PGIS induced in fibroblasts under hypoxic condition. Since PGIS is involved in the enhanced expression of VEGF under hypoxic condition in human fibroblasts, PGIS may be a novel target for antitumor angiogenesis therapy.

\section{Acknowledgements}

We thank Professor Akio Tomoda of Tokyo Medical University for providing NB-1 cells; Professor Shoji Natsugoe of Kagoshima University for human coloncancer paraffin block; Dr Hiroshi Shirahama, Dr Yukie Tashiro, Mr. Keishi Tokunagana, Ms. Yasuko Shinmura of Imakiire General Hospital for preparing good paraffin sections from the archival paraffin block specimens; and Ms. Hiromi Mitsuo and Ms. Naomi Nakanishi for valuable secretarial assistance.

\section{References}

1. Nakagawa H, Liyanarachchi S, Davuluri RV, Auer H Martin EW Jr, de la Chapelle A and Frankel WL: Role of cancer-associated stromal fibroblasts in metastatic colon cancer to the liver and their expression profiles. Oncogene 23 7366-7377, 2004.

2. Fidler IJ: The pathogenesis of cancer metastasis: the 'seed and soil' hypothesis revisited. Nat Rev Cancer 3: 453-458, 2003.

3. Cutler NS, Graves-Deal R, LaFleur BJ, Gao Z, Boman BM, Whitehead RH, Terry E, Morrow JD and Coffey RJ: Stromal production of prostacyclin confers an antiapoptotic effect to colonic epithelial cells. Cancer Res 63: 1748-1751, 2003.

4. Ingerman-Wojenski C, Silver MJ, Smith JB and Macarak E: Bovine endothelial cells in culture produce thromboxane as well as prostacyclin. J Clin Invest 67: 1292-1296, 1981.

5. Moncada S, Gryglewski R, Bunting S and Vane JR: An enzyme isolated from arteries transforms prostaglandin endoperoxides to an unstable substance that inhibits platelet aggregation. Nature 263: 663-665, 1976.

6. Moncada S, Herman AG, Higgs EA and Vane JR: Differential formation of prostacyclin (PGX or PGI2) by layers of the arterial wall. An explanation for the anti-thrombotic properties of vascular endothelium. Thromb Res 11: 323-344, 1977.

7. Ullrich V, Castle L and Weber P: Spectral evidence for the cytochrome P450 nature of prostacyclin synthetase. Biochem Pharmacol 30: 2033-2036, 1981.

8. Weksler BB, Ley CW and Jaffe EA: Stimulation of endothelial cell prostacyclin production by thrombin, trypsin, and the ionophore A 23187. J Clin Invest 62: 923-930, 1978.

9. Yokoyama C, Yabuki T, Shimonishi M, Wada M, Hatae T, Ohkawara S, Takeda J, Kinoshita T, Okabe M and Tanabe T: Prostacyclin-deficient mice develop ischemic renal disorders, including nephrosclerosis and renal infarction. Circulation 106: 2397-2403, 2002.
10. Gupta RA, Tan J, Krause WF, Geraci MW, Willson TM, Dey SK and DuBois RN: Prostacyclin-mediated activation of peroxisome proliferator-activated receptor delta in colorectal cancer. Proc Natl Acad Sci USA 97: 13275-13280, 2000.

11. Wu KK and Liou JY: Cyclooxygenase inhibitors induce colon cancer cell apoptosis via PPARdelta $\rightarrow$ 14-3-3epsilon pathway. Methods Mol Biol 512: 295-307, 2009.

12. Arany Z, Foo SY, Ma Y, Ruas JL, Bommi-Reddy A, Girnun G, Cooper M, Laznik D, Chinsomboon J, Rangwala SM, Baek KH, Rosenzweig A and Spiegelman BM: HIF-independent regulation of VEGF and angiogenesis by the transcriptional coactivator PGC-1alpha. Nature 451: 1008-1012, 2008.

13. Mizukami Y, Li J, Zhang X, Zimmer MA, Iliopoulos O and Chung DC: Hypoxia-inducible factor-1-independent regulation of vascular endothelial growth factor by hypoxia in colon cancer. Cancer Res 64: 1765-1772, 2004.

14. Wang J, Hasui K, Jia X, Matsuyama T and Eizuru Y: Possible role for external environmental stimuli in nasopharyngeal NK/T-cell lymphomas in the northeast of China with EBV infection-related autophagic cell death: a pathoepidemiological analysis. J Clin Exp Hematop 49: 97-108, 2009.

15. Nakajima Y, Gotanda T, Uchimiya H, Furukawa T, Haraguchi M, Ikeda R, Sumizawa T, Yoshida $\mathrm{H}$ and Akiyama S: Inhibition of metastasis of tumor cells overexpressing thymidine phosphorylase by 2-deoxy-L-ribose. Cancer Res 64: 1794-1801, 2004.

16. Buchanan FG, Chang W, Sheng H, Shao J, Morrow JD and DuBois RN: Up-regulation of the enzymes involved in prostacyclin synthesis via Ras induces vascular endothelial growth factor. Gastroenterology 127: 1391-1400, 2004

17. Kamio K, Sato T, Liu X, Sugiura H, Togo S, Kobayashi T, Kawasaki S, Wang X, Mao L, Ahn Y, Holz O, Magnussen H and Rennard SI: Prostacyclin analogs stimulate VEGF production from human lung fibroblasts in culture. Am J Physiol Lung Cell Mol Physiol 294: L1226-L1232, 2008.

18. Tong BJ, Tan J, Tajeda L, Das SK, Chapman JA, DuBois RN and Dey SK: Heightened expression of cyclooxygenase- 2 and peroxisome proliferator-activated receptor-delta in human endometrial adenocarcinoma. Neoplasia 2: 483-490, 2000.

19. Piqueras L, Reynolds AR, Hodivala-Dilke KM, Alfranca A, Redondo JM, Hatae T, Tanabe T, Warner TD and Bishop-Bailey D: Activation of PPARbeta/delta induces endothelial cell proliferation and angiogenesis. Arterioscler Thromb Vasc Biol 27: 63-69, 2007.

20. Moreira IS, Fernandes PA and Ramos MJ: Vascular endothelial growth factor (VEGF) inhibition - a critical review. Anticancer Agents Med Chem 7: 223-245, 2007.

21. Wang D, Wang H, Guo Y, Ning W, Katkuri S, Wahli W, Desvergne B, Dey SK and DuBois RN: Crosstalk between peroxisome proliferator-activated receptor delta and VEGF stimulates cancer progression. Proc Natl Acad Sci USA 103: 19069-19074, 2006.

22. Fukumoto K, Yano Y, Virgona N, Hagiwara H, Sato H, Senba H, Suzuki K, Asano R, Yamada K and Yano T: Peroxisome proliferator-activated receptor delta as a molecular target to regulate lung cancer cell growth. FEBS Lett 579: 3829-3836, 2005.

23. Camacho M, Rodríguez C, Guadall A, Alcolea S, Orriols M, Escudero JR, Martínez-González J and Vila L: Hypoxia upregulates PGI-synthase and increases PGI release in human vascular cells exposed to inflammatory stimuli. J Lipid Res 52: 720-731, 2011. 